\title{
The Importance of Solid-state Molecular Motion to Room Temperature Phosphorescence
}

Yujie Tu, Junkai Liu, Xuepeng Zhang, Tsz, Shing Cheung, Xuewen He, Jing Guo, Jacky W.Y. Lam, Sijie Chen, * and Ben Zhong Tang*

Dr. Y. Tu, J. Liu, Dr. X. Zhang, T.S.Cheung, Dr. X. He, Prof. J.W.Y.Lam, Prof. B.Z.Tang. Department of Chemistry, Hong Kong Branch of Chinese National Engineering Research Center for Tissue Restoration and Reconstruction, State Key Laboratory of Neuroscience, Department of Chemical and Biological Engineering, and Division of Life Science, The Hong Kong University of Science and Technology, Clear Water Bay, Kowloon, 999077, Hong Kong, China. Center for Aggregation-Induced Emission, SCUT-HKUST Joint Research Institute, State Key Laboratory of Luminescent Materials and Devices, South China University of Technology, Guangzhou, 510640, China. E-mail: tangbenz@ust.hk

Dr. S. Chen, Prof. B.Z.Tang. Ming Wai Lau Centre for Reparative Medicine, Karolinska Institutet, Hong Kong. E-mail: sijie.chen@ki.se

Prof. B.Z.Tang, AIE Institute, Guangzhou Development District, Huangpu, Guangzhou 510530, China.

J. Guo. State Key Laboratory of Chemo/Biosensing and Chemometrics, College of Chemistry and Chemical Engineering, Hunan University, Changsha 410082, China.

Keywords: room temperature phosphorescence, spin-vibronic coupling, solid-state molecular motion.

\footnotetext{
Abstract: Molecular motion is often considered detrimental to luminescence because it favors nonradiative decay. However, nothing is absolute, and molecular motion can also do useful work if utilized properly. For example, photothermal therapy makes use of the heat generated in light irradiation for cancer treatment. To further explore the merits of molecular motion, ortho-substituted benzoic acids were used as model compounds to evaluate the importance of molecular motion to luminescence in the solid state. It is verified that the twisting of the carboxylic acid group can activate spin vibronic coupling to facilitate intersystem crossing to result in more efficient room temperature phosphorescence (RTP). A five-state model is established to understand the ISC process and an effective pre-twisted molecular design strategy is put forward for the development of efficient RTP materials.
} 
Molecular motion is commonly known to be detrimental to luminescence because it promotes nonradiative decay accompanied by the transformation of optical energy to other forms such as thermal energy. Thus, scientists attempt to avoid molecular motion and maximize the emission by restriction of molecular motion. ${ }^{1}$ In recent years, there has been interest in utilizing molecular motions generated by photo-irradiation to perform useful applications. For example, the photothermal effect of aggregation-induced emission (AIE) luminogens has been intensively explored for effective cancer therapy, ${ }^{2}$ and the associated photoacoustic effect can be used for deep in-vivo imaging. ${ }^{3}$ Therefore, nothing is absolute and molecular motion is sometimes useful, depending on how we utilize it.

Molecular motion can be applied in the biomedical area and also can be utilized to manipulate luminescence as well. ${ }^{4}$ In the solid state, molecular motion can tune the intermolecular interaction and gives rise to packing or morphology change. For example, some luminogens can spontaneously form crystals after the thermal acceleration of molecular motions and cooling down. And if their light emission can be turned on upon the crystal formation, such a phenomenon is known as crystallization-induced emission. ${ }^{5}$ In contrast, the light can be completely quenched after crystallization due to the formation of extensive $\pi-\pi$ interactions ${ }^{6}$ or the activation of intersystem crossing (ISC) to triplet states which serves as dark states to quench fluorescence ${ }^{7}$. Although ISC is a non-radiative pathway for fluorescence, it is a prerequisite for phosphorescence. Therefore, rather than fighting against the nonradiative decay facilitated by molecular motion, it's better to utilize it to activate efficient ISC and achieve room temperature phosphorescence (RTP) since RTP materials can be widely applied in fields including OLED, anti-counterfeiting, bioimaging, etc. ${ }^{8}$

In the previous studies, scientists usually consider the direct spin-orbit coupling (SOC) as the driving force of the "spin-forbidden" ISC process, in which only the pure electronic character matters and nuclear motions are not concerned. However, the second-order spin-orbit coupling 
which involves the vibronic coupling (VC) between electronic states driven by molecular motions is often underestimated even ignored because of some simple and restrictive assumptions. For example, VC which accounts for internal conversion (IC) and SOC which accounts for ISC are regarded as two separate processes due to their vastly different time scale. As a matter of fact, VC and SOC will occur simultaneously in many molecular systems with flexible nuclear motions. Therefore, we need to consider the mechanism of molecular motionmediated spin-vibronic coupling (SVC) which means simultaneous SOC with VC in the singlet/triplet manifolds. ${ }^{9}$

To evaluate whether the molecular motion and SVC mechanism can facilitate ISC and RTP, we attempt to construct simple pure organic RTP molecules with varied freedom of motions as model compounds according to the well-accepted design principle of combining a $\pi$ platform with a carbonyl moiety in one molecule. ${ }^{10}$ Benzoic acid was chosen as the ideal parent form of the model compounds. ${ }^{11}$ The methoxy group which can enhance ISC and RTP ${ }^{12}$ is introduced to change the conjugation, generate steric effects, and tune the freedom of molecular motion.

Benzoic acid (BA) shows negligible light emission and a relatively short RTP lifetime (i.e. $0.42 \mathrm{~ms}$ ). By varying the substitution position of methoxy groups, we found that 2-methoxy benzoic acid (oMOBA) exhibits a longer RTP lifetime than the meta- or para-substituted ones ( $m \mathrm{MOBA}$ and $p \mathrm{MOBA}$ ). If both the ortho positions are methoxylated, the resulting 2,6dimethoxy benzoic acid (oDMOBA) displays an even ultralong RTP lifetime up to $746 \mathrm{~ms}$ with a visible afterglow longer than 10 seconds. The ortho substituents are believed to introduce steric hindrance to twist the carboxylic acid (CA) group. The CA twisting angles $(\theta)$ to phenyl rings of the five compounds in crystals follow the order: $\mathrm{BA}<p \mathrm{MOBA}<m \mathrm{MOBA}<o \mathrm{MOBA}$ $<o$ DMOBA. Apparently, there is a positive correlation between $\theta$ and RTP lifetime (Fig. 1A and $1 \mathrm{~B})$. And it is natural to hypothesize that "the larger the CA twisting, the better is the RTP performance". 
To prove such a claim, the photophysical properties of less twisted $o$ MOBA and highly twisted $o$ DMOBA are further studied. $o$ MOBA and $o$ DMOBA show strong absorption peaked at $295 \mathrm{~nm}$ and $275 \mathrm{~nm}$, respectively in solution (Fig. S1). We measured the photoluminescence spectra of their crystals at an excitation of $285 \mathrm{~nm}$. Both molecules display similar dual emission consisting of delayed fluorescence (DF) at $\sim 360 \mathrm{~nm}$ and RTP at $\sim 500 \mathrm{~nm}$ (Fig. 1C, 1D and S2). To compare the RTP performance, their quantum yield and average lifetime are measured at excitation wavelengths of not only $285 \mathrm{~nm}$ but also $365 \mathrm{~nm}$ to exclude the influence of DF (Fig. S3 and Table S1). Results suggest that $o$ DMOBA exhibits higher brightness and longer lifetime than $o$ MOBA.

Meanwhile, the $\theta$ of $o$ DMOBA $\left(55.3^{\circ}\right)$ is obviously much larger than $o$ MOBA $\left(5.80^{\circ}\right)$ according to their crystal structures (Fig. 2). oMOBA crystal exhibits a layer-by-layer packing. Within each layer, two $o$ MOBA molecules display a head-to-head dimeric packing joined by two intermolecular hydrogen bonds between their CA moieties. Other compounds, namely BA, $m \mathrm{MOBA}$, and $p \mathrm{MOBA}$ exhibit a similar packing mode (Fig. S4). In contrast, it is difficult for $o$ DMOBA molecule to adopt a planar conformation due to the steric effect of the two orthosubstituents. The twisted CA group of each oDMOBA molecule forms two hydrogen bonds with two neighbor molecules hand-by-hand. It seems that a chain of molecules is adhered together as a "polymer" by continuous hydrogen bonds. Indeed, the crystal packing makes a difference to RTP as reported in previous works. ${ }^{13}$ Rather than the $o$ MOBA's dimeric packing where the CA group is rigidly constrained to be "coplanar" to the phenyl ring. The unique crystal packing of $o$ DMOBA maintains the CA group in a twisted manner and endows $o$ DMOBA molecules with more freedom of molecular rotation.

To examine the role of $\theta$ and the importance of molecular motion, the potential energy surfaces (PESs) of $o$ DMOBA along the CA twisting are calculated. As shown in Fig. 3A, the first two excited states of singlet and triplet manifolds are identified to be ${ }^{1}\left(\pi, \pi^{*}\right),{ }^{1}\left(\mathrm{n}, \pi^{*}\right)$, ${ }^{3}\left(\pi, \pi^{*}\right)$, and ${ }^{3}\left(\mathrm{n}, \pi^{*}\right)$ according to their electronic transition characters. From the PESs, it is easy 
to recognize that there are ${ }^{1}\left(\pi, \pi^{*}\right)-^{1}\left(n, \pi^{*}\right)$ and ${ }^{3}\left(\pi, \pi^{*}\right)-{ }^{3}\left(n, \pi^{*}\right)$ crossing at $\theta$ from $50^{\circ}$ to $40^{\circ}$, indicating strong vibronic interactions of $\left(\pi, \pi^{*}\right)$ and $\left(\mathrm{n}, \pi^{*}\right)$ states. ${ }^{14}$ We can better understand the $\left(\pi, \pi^{*}\right)-\left(\mathrm{n}, \pi^{*}\right)$ vibronic coupling by the frontier molecular orbitals at $\theta$ of $50^{\circ}$ and $40^{\circ}$ (Fig. 3B and Table S3). The HOMO at $50^{\circ}$ which is $\pi$ orbital in character shifts to HOMO-2 at $40^{\circ}$, while the HOMO-2 at $50^{\circ}$ which is n orbital in character shifts to $\mathrm{HOMO}$ at $40^{\circ}$. In brief, there is a $\mathrm{n}-\pi \mathrm{MO}$ ordering reversal from $50^{\circ}$ to $40^{\circ}$. As analyzed in Fig. $3 \mathrm{C}$, when the CA group is twisted, the $\pi$ electrons of the CA group are not delocalized in the phenyl ring, yet the lone pairs of carbonyl oxygen are partially overlapped with the $\pi$ plane. After CA twisting, $\pi$ electrons of the CA group is more involved in the $\pi$ conjugation, whereas the lone pairs become more or less orthogonal to the $\pi$ plane, which result in the stabilization of the $\pi$ orbital and destabilization of the $\mathrm{n}$ orbital. Therefore, the CA twisting serves as a driving force of the $n-\pi$ ordering reversal and it couples the $\left(\pi, \pi^{*}\right)$ and $\left(n, \pi^{*}\right)$ excited states vibronically.

According to the El-Sayed rule, ISC is allowed between ${ }^{1}\left(\pi, \pi^{*}\right)$ and ${ }^{3}\left(n, \pi^{*}\right)$, or between ${ }^{1}\left(\mathrm{n}, \pi^{*}\right)$ and ${ }^{3}\left(\pi, \pi^{*}\right) \cdot{ }^{15}$ If we only considered a fixed molecular geometry, the driving force of ISC is the direct SOC based on the pure electronic characters. However, molecular motion, which refers to the CA twisting in the $o$ DMOBA system, is also an important driving force of ISC. Spin flipping is much likely to occur simultaneously with $\left(\pi, \pi^{*}\right)-\left(\mathrm{n}, \pi^{*}\right)$ vibronic coupling. In this regard, the SVC mechanism is more than meaningful for efficient ISC.

From the PESs of Fig. 3A, another point is worth to be noticed. At $\theta$ from $40^{\circ}$ to $10^{\circ}$, the ${ }^{3}\left(\pi, \pi^{*}\right)$ and ${ }^{3}\left(n, \pi^{*}\right)$ states are nearly degenerate and mixed in different ${ }^{3}\left(\pi, \pi^{*}\right)$ and ${ }^{3}\left(n, \pi^{*}\right)$ proportions (Fig. 3D). For example, at $40^{\circ}$, the lowest two triplet states are both admixtures of nearly half ${ }^{3}\left(\mathrm{n}, \pi^{*}\right)$ transition from HOMO to LUMO and half ${ }^{3}\left(\pi, \pi^{*}\right)$ transition from HOMO-1 to LUMO (Fig. 3E). We can understand such transition hybridization from the perspective of either schematic PESs along a nuclear coordinate or an energy level diagram at a fixed geometry (Fig. 3F). Since ${ }^{3}\left(\pi, \pi^{*}\right)$ state contributes to RTP with short brightness and long lifetime whereas ${ }^{3}\left(\mathrm{n}, \pi^{*}\right)$ state gives rise to RTP with high brightness and short lifetime, the transition 
hybridization caused by the strong ${ }^{3}\left(\pi, \pi^{*}\right)-^{3}\left(\mathrm{n}, \pi^{*}\right)$ vibronic coupling is useful to obtain a good balance between brightness and lifetime to achieve both efficient and persistent RTP emitters. ${ }^{10 a}$, 16

In summary, from the PESs of $o$ DMOBA along the CA twisting coordinate, we found that molecular motion gives rise to $\left(\pi, \pi^{*}\right)-\left(\mathrm{n}, \pi^{*}\right)$ vibronic coupling, which not only favors ISC due to the SVC mechanism but also helps to achieve good RTP performance with balanced brightness and lifetime. Previously, it is a common statement that crystallization is an ideal tool to enhance RTP due to the prevention of air penetration to reduce the emission quenching by oxygen and the restriction of molecular motion to suppress the non-radiative decay. ${ }^{17}$ However, as we demonstrated, not all molecular motions always do harm to luminescence. Instead, a certain molecular motion which specifically couples $\left(\pi, \pi^{*}\right)$ and $\left(\mathrm{n}, \pi^{*}\right)$ states is beneficial to efficient ISC and RTP.

To extend the conclusion from the isolated molecule to the solid-state molecule, we performed simulations on both $o$ MOBA and $o$ DMOBA crystals. In the previous reports, the normal two-layer QM/MM model was often applied, in which the effect of hydrogen bonds was underestimated. In this work, a seldom reported three-layer High QM / medium QM / low MM model was adopted to fully estimate the significant role of hydrogen bonds formed by the neighbor molecules (Fig. 4A and 4E). For example, the real $\theta$ value of the ground state of $o$ DMOBA crystal should be $55.3^{\circ}$. The calculated $\theta$ values of the optimized ground state of $o$ DMOBA crystal by adopting two-layer or three-layer models are $14.3^{\circ}$ and $52.3^{\circ}$, respectively (Fig. S5). Therefore, the three-layer model was proved to be more accurate than the two-layer model, indicating that it is a good approach for simulations with strong intermolecular interactions. The ground state and the first two excited states of singlet and triplet manifolds of $o$ MOBA (Table S5) and $o$ DMOBA (Table S7) are optimized. To evaluate the effect of the CA twisting in the solid state, we extract the energy level diagrams of $o$ MOBA and $o$ DMOBA at geometries of $S_{0, \min }$ where the CA group is more twisted (Fig. $4 \mathrm{~B}$ and $4 \mathrm{~F}$ ) and $\mathrm{S}_{1, \mathrm{~min}}$ where the 
CA group is less twisted (Fig. 4C and 4G). The schematic PESs of their ground state and the four excited states are plotted approximately based on the adiabatic excitation energies ( $\mathrm{Y}$-axis) and the $\theta$ values (X-axis) of each excited state minimums (Fig. 4D and 4H).

For both $o \mathrm{MOBA}$ and $o \mathrm{DMOBA}$, the CA groups tend to rotate and become less twisted after excitation, and energies of $\left(n, \pi^{*}\right)$ states in either singlet or triplet manifolds become lower (Fig. 4B $\rightarrow$ 4C and Fig. 4F $\rightarrow$ 4G). However, their distinctions are obvious: 1) The excited-state molecular motion of $o$ DMOBA $\left(\theta_{1}: 52.3^{\circ} \rightarrow \theta_{2}: 17.8^{\circ}\right)$ is in larger amplitude than that of $o$ MOBA $\left(\theta_{1}: 7.4^{\circ} \rightarrow \theta_{2}:-0.9^{\circ}\right)$. The crossing of $\left(\mathrm{n}, \pi^{*}\right)$ and $\left(\pi, \pi^{*}\right)$ states at a certain $\theta$ between $\theta_{1}$ and $\theta_{2}$ should be more likely to occur in $o$ DMOBA. 2) the ${ }^{3}\left(\mathrm{n}, \pi^{*}\right)$ state and ${ }^{3}\left(\pi, \pi^{*}\right)$ state are hybridized in $o$ DMOBA but discrete in $o$ MOBA. 3) The HOMO of $o$ MOBA crystal in each state is always a $\pi$ orbital, whereas the $n-\pi$ orbital ordering reversal does happen in $o$ DMOBA crystal. The HOMO is a $\pi$ orbital in twisted conformations but becomes a $\mathrm{n}$ orbital in less twisted conformations (Table S4 and S6). 4) The optimized $T_{1}$ is ${ }^{3}\left(\pi, \pi^{*}\right)$ state and $T_{2}$ is ${ }^{3}\left(n, \pi^{*}\right)$ state for $o$ DMOBA crystal, but the optimized $\mathrm{T}_{1} / \mathrm{T}_{2}$ are both ${ }^{3}\left(\pi, \pi^{*}\right)$ states for $o$ MOBA crystal (Fig. 4D and 4G, Table S5 and S7). Based on these significant differences, it is noticeable that the more remarkable molecular motion in $o$ DMOBA results in more manifest $\left(\pi, \pi^{*}\right) \leftrightarrow\left(\mathrm{n}, \pi^{*}\right)$ transitions, thus more significant SVC, more efficient ISC, and RTP with better performance.

All in all, from excitation to emission, the CA group changes its conformation from twisted to less twisted and then twisted again. Such molecular motions drive the ISC processes $\left(\mathrm{S}_{1} \rightarrow \mathrm{T}_{\mathrm{n}}\right.$, $\mathrm{T}_{1} \rightarrow \mathrm{S}_{0}$ ) via the SVC mechanism. To better understand it, we can uncover the black box of photophysical processes of $o$ DMOBA from the perspective of frontier molecular orbital diagrams (Fig. 5A). After ( $\left.\pi, \pi^{*}\right)$ excitation, the molecule tends to relax to a lower energy state to adopt a more planar conformation. As we mentioned, planarization of the CA group towards the phenyl ring will decrease the $n-\pi$ overlap and increase the $\pi$ conjugation of the CA group. This raises the energy of the $n$ orbital but drops the $\pi$ orbital energy. Thus, the $n$ and $\pi$ orbitals become degenerate at a certain $\theta$ value at which the n-to- $\pi$ electron transfer occurs. The half- 
filled $\pi$ orbital becomes full and stabilized, while the $\mathrm{n}$ orbital becomes half-filled and destabilized. The overall effect is the $n-\pi$ MO reversal and $\left(\pi, \pi^{*}\right)-\left(n, \pi^{*}\right)$ vibronic coupling. If the spin remains unchanged during the electron transfer, the process is just internal conversion (IC). However, if a spin flipping simultaneously occurs with the vibronic coupling, the process is now ISC which is facilitated by SVC.

So far, we have comprehensively studied the importance of molecular motion of $o$ DMOBA system in the solid state. In fact, oDMOBA molecule is nothing special, and the SVC mechanism and molecular-motion-facilitated ISC are supposed to participate in most RTP systems especially for heteroatom-containing RTP molecules. Herein, we establish a simplified five-state model consisting of $\mathrm{S}_{0},{ }^{1}\left(\pi, \pi^{*}\right),{ }^{1}\left(\mathrm{n}, \pi^{*}\right),{ }^{3}\left(\pi, \pi^{*}\right),{ }^{3}\left(\mathrm{n}, \pi^{*}\right)$ to explain the RTP systems in which certain molecular motion couples the $\left(\pi, \pi^{*}\right)$ and $\left(\mathrm{n}, \pi^{*}\right)$ states and favors the ISC and RTP (Fig. 5B). In this work, the CA twisting is the switch between $\left(\pi, \pi^{*}\right)$ and $\left(n, \pi^{*}\right)$ states. The steric effect of the ortho-substituents and intermolecular hydrogen bonds orient the CA group to pre-twist in the crystal, endowing the CA group with a bigger amplitude of molecular motion after excitation. Yet the CA groups of other benzoic acid derivatives with smaller $\theta$ have less room to rotate. Based on this work, it is natural for us to conclude a rational design strategy, namely pre-twisting the heteroatom-containing group, especially the carbonyl group to allow the occurrence of the pivotal molecular motion that couples $\left(\pi, \pi^{*}\right)$ and $\left(n, \pi^{*}\right)$ states. The new strategy provides us a new guideline and broadens our horizons to design more RTP materials with good performance. As a perspective, there remains several important issues to be further addressed in this molecular system such as to experimentally investigate the excited-state processes and to evaluate the electronic substitution effect of methoxy group by replacing it with other substituents (e.g. methyl group).

In conclusion, we verified the importance of molecular motions to RTP in the solid state, demonstrated the significance of the SVC mechanism to the RTP research, established a fivestate model to understand the ISC process and put forward an effective pre-twisted molecular 
design strategy for the further development of efficient RTP materials. More importantly, we have learned that a new viewpoint often emerges from trials to utilize something bad. Instead of fighting against the molecular motion all the time, we can explore the merits of molecular motion in different ways in future research.

\section{Supporting Information}

Supporting Information is available from the Wiley Online Library or from the author.

\section{Acknowledgements}

We are grateful for financial support from the National Science Foundation of China (21788102 and 81501591), the Research Grants of Council of Hong Kong (16305518,16305618, N-HKUST609/19, A-HKUST605/16, and C6009-17G), the National Key Research and Development Program of China (2018YFE 0190200), the Innovation of Technology Commission (ITC-CNERC14SC01), and Ming Wai Lau Centre for Reparative Medicine Associate Member Programme (MWLC19SC02).

Yujie Tu and Junkai Liu contributed equally to this work.

\section{Conflict of interest}

The authors declare no conflict of interest.

\section{References}

[1] J. Mei, N. L. C. Leung, R. T. K. Kwok, J. W. Y. Lam, B. Z. Tang, Chem. Rev. 2015, 115, 11718.

[2] a) Z. Zhao, C. Chen, W. T. Wu, F. F. Wang, L. L. Du, X. Y. Zhang, Y. Xiong, X. W. He, Y. J. Cai, R. T. K. Kwok, J. W. Y. Lam, X. K. Gao, P. C. Sun, D. L. Phillips, D. Ding, B. Z. Tang, Nat. Commun. 2019, 10; b) S. J. Liu, X. Zhou, H. K. Zhang, H. L. Ou, J. W. Y. 
Lam, Y. Liu, L. Q. Shi, D. Ding, B. Z. Tang, J. Am. Chem. Soc. 2019, 141, 5359; c) D. Wang, M. M. S. Lee, W. H. Xu, G. G. Shan, X. Y. Zheng, R. T. K. Kwok, J. W. Y. Lam, X. L. Hu, B. Z. Tang, Angew. Chem. Int. Ed. 2019, 58, 5628.

[3] a) J. Geng, L. D. Liao, W. Qin, B. Z. Tang, N. Thakor, B. Liu, J. Nanosci. Nanotechnol. 2015, 15, 1864; b) Z. H. Sheng, B. Guo, D. H. Hu, S. D. Xu, W. B. Wu, W. H. Liew, K. Yao, J. Y. Jiang, C. B. Liu, H. R. Zheng, B. Liu, Adv. Mater. 2018, 30.

[4] S. Liu, Y. Li, H. Zhang, Z. Zhao, X. Lu, J. W. Y. Lam, B. Z. Tang, ACS Mater. Lett. 2019, $1,425$.

[5] X. L. Luo, J. N. Li, C. H. Li, L. P. Heng, Y. Q. Dong, Z. P. Liu, Z. S. Bo, B. Z. Tang, Adv. Mater. 2011, 23, 3261.

[6] P. Alam, N. L. C. Leung, Y. H. Cheng, H. K. Zhang, J. K. Liu, W. J. Wu, R. T. K. Kwok, J. W. Y. Lam, H. H. Y. Sung, I. D. Williams, B. Z. Tang, Angew. Chem. Int. Ed. 2019, 58, 4536.

[7] W. J. Zhao, Z. K. He, Q. Peng, J. W. Y. Lam, H. L. Ma, Z. J. Qiu, Y. C. Chen, Z. Zhao, Z. G. Shuai, Y. Q. Dong, B. Z. Tang, Nat. Commun. 2018, 9.

[8] a) S. Xu, R. F. Chen, C. Zheng, W. Huang, Adv. Mater. 2016, 28, 9920; b) J. H. Zhi, Q. Zhou, H. F. Shi, Z. F. An, W. Huang, Chem. Asian J. 2020, 15, 947.

[9] a) T. J. Penfold, E. Gindensperger, C. Daniel, C. M. Marian, Chem. Rev. 2018, 118, 6975; b) R. Huang, J. Avó, T. Northey, E. Chaning-Pearce, P. L. dos Santos, J. S. Ward, P. Data, M. K. Etherington, M. A. Fox, T. J. Penfold, M. N. Berberan-Santos, J. C. Lima, M. R. Bryce, F. B. Dias, J. Mater. Chem. C. 2017, 5, 6269; c) H. Bhatia, D. Ray, Mater. Adv. 2020; d) T. Serevičius, T. Bučiūnas, J. Bucevičius, J. Dodonova, S. Tumkevičius, K. Kazlauskas, S. Juršènas, J. Mater. Chem. C. 2018, 6, 11128.

[10] a) W. J. Zhao, Z. K. He, J. W. Y. Lam, Q. Peng, H. L. Ma, Z. G. Shuai, G. X. Bai, J. H. Hao, B. Z. Tang, Chem 2016, 1, 592; b) Z. Y. Yang, Z. Mao, X. P. Zhang, D. P. Ou, Y. X. Mu, Y. Zhang, C. Y. Zhao, S. W. Liu, Z. G. Chi, J. R. Xu, Y. C. Wu, P. Y. Lu, A. Lien, 
M. R. Bryce, Angew. Chem. Int. Ed. 2016, 55, 2181.

[11] a) Y. Gong, L. Zhao, Q. Peng, D. Fan, W. Z. Yuan, Y. Zhang, B. Z. Tang, Chem. Sci. 2015, 6, 4438; b) Z. C. Cheng, H. F. Shi, H. L. Ma, L. F. Bian, Q. Wu, L. Gu, S. Z. Cai, X. Wang, W. W. Xiong, Z. F. An, W. Huang, Angew. Chem. Int. Ed. 2018, 57, 678.

[12] a) J. S. Ward, R. S. Nobuyasu, M. A. Fox, J. A. Aguilar, D. Hall, A. S. Batsanov, Z. J. Ren, F. B. Dias, M. R. Bryce, J. Org. Chem. 2019, 84, 3801; b) Z. Y. Yu, Y. S. Wu, Q. Peng, C. L. Sun, J. W. Chen, J. N. Yao, H. B. Fu, Chem. Eur. J. 2016, 22, 4717; c) B. Chen, X. P. Zhang, Y. C. Wang, H. Miao, G. Q. Zhang, Chem. Asian J. 2019, 14, 751; d) C. A. DeRosa, M. L. Daly, C. Kerr, C. L. Fraser, Chemphotochem 2019, 3, 31.

[13] a) J. Yang, X. Zhen, B. Wang, X. M. Gao, Z. C. Ren, J. Q. Wang, Y. J. Xie, J. R. Li, Q. Peng, K. Y. Pu, Z. Li, Nat. Commun. 2018, 9; b) Z. F. Chai, C. Wang, J. F. Wang, F. Liu, Y. J. Xie, Y. Z. Zhang, J. R. Li, Q. Q. Li, Z. Li, Chem.Sci. 2017, 8, 8336.

[14] Y. J. Tu, J. K. Liu, H. K. Zhang, Q. Peng, J. W. Y. Lam, B. Z. Tang, Angew. Chem. Int. Ed. 2019, 58, 14911.

[15] S. Lower, M. El-Sayed, Chem. Rev. 1966, 66, 199.

[16] a) H. Ma, Q. Peng, Z. An, W. Huang, Z. Shuai, J. Am. Chem. Soc. 2019, 141, 1010; b) J. R. Chen, N. U. Rahman, Z. Mao, J. Zhao, Z. Y. Yang, S. W. Liu, Y. Zhang, Z. G. Chi, J. Mater. Chem. C. 2019, 7, 8250.

[17] W. Z. Yuan, X. Y. Shen, H. Zhao, J. W. Y. Lam, L. Tang, P. Lu, C. L. Wang, Y. Liu, Z. M. Wang, Q. Zheng, J. Z. Sun, Y. G. Ma, B. Z. Tang, J. Phys. Chem. C. 2010, 114, 6090. 
A
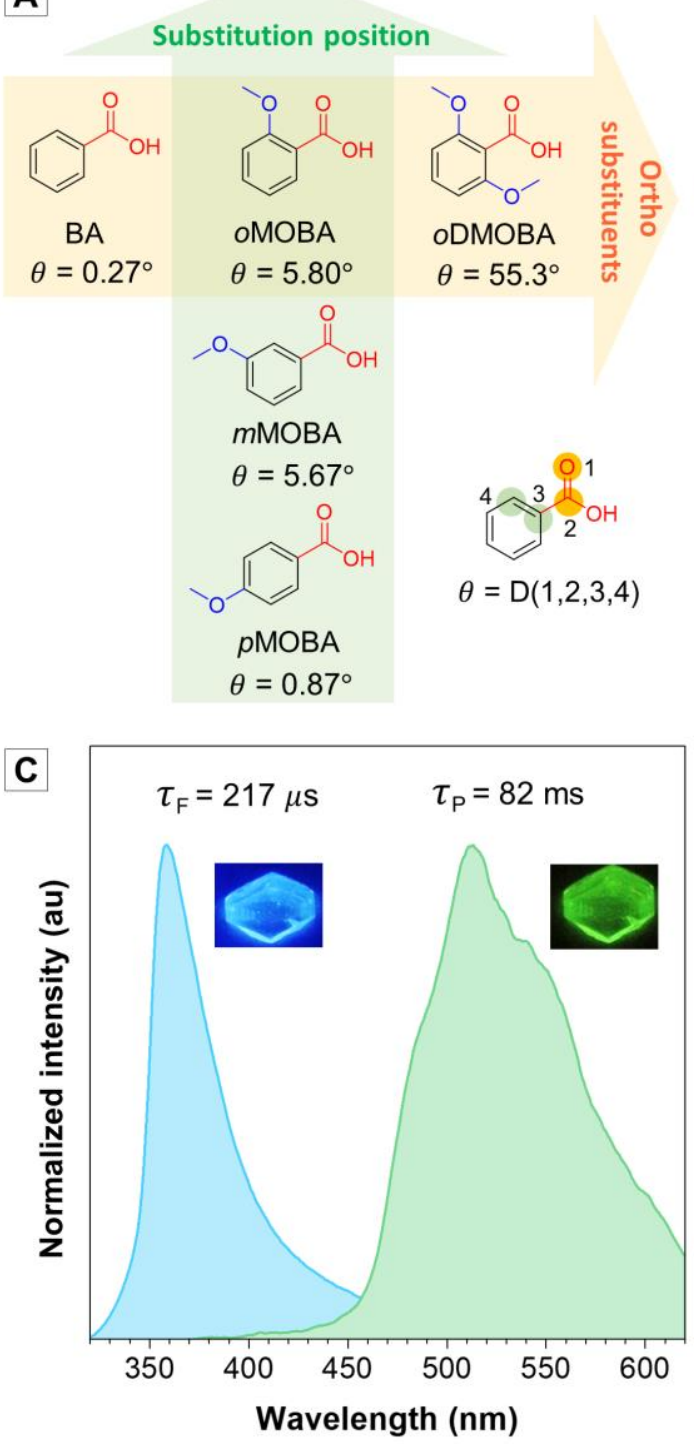

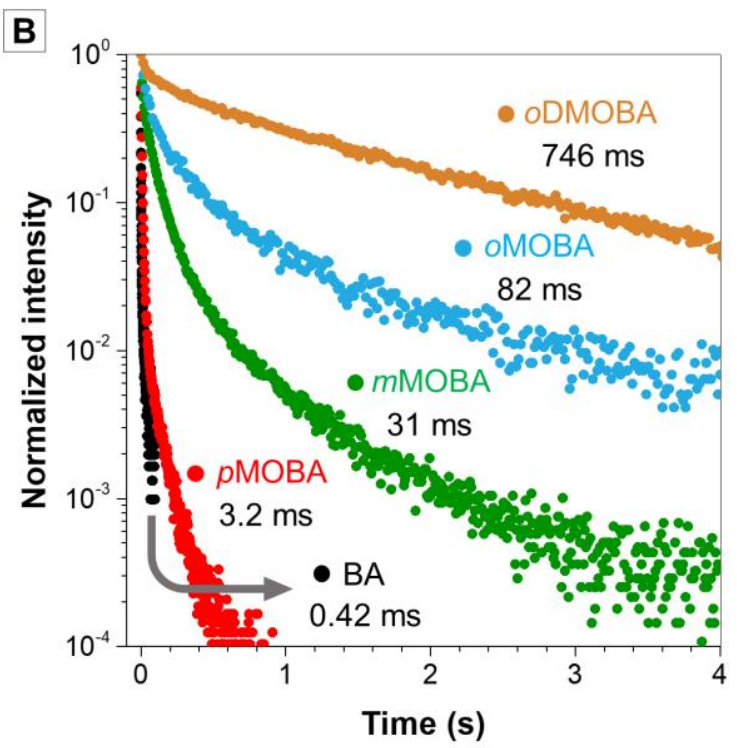

D

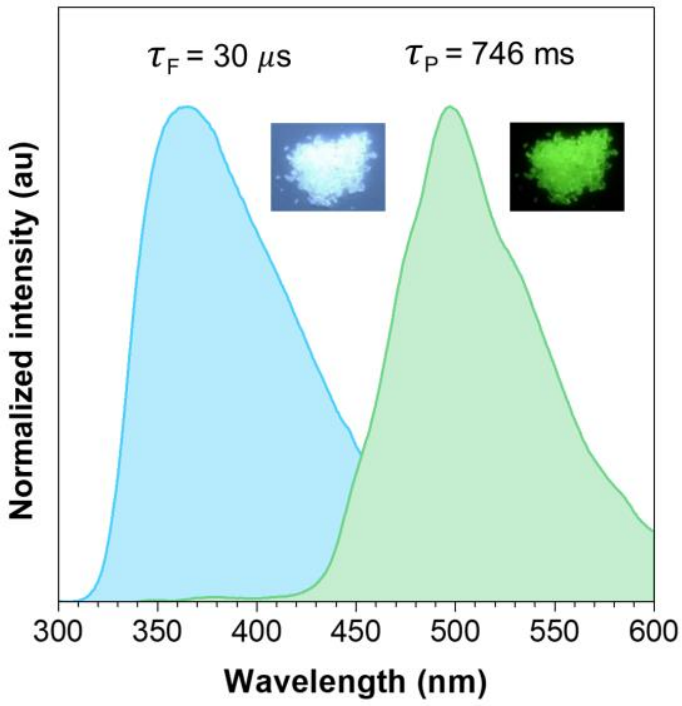

Figure 1. (A) Benzoic acid derivatives with varied substitution positions and substituent numbers. $\theta$ represents the dihedral angle between the carboxylic acid (CA) plane and the Phenyl (Ph) plane, namely $\mathrm{D}(1,2,3,4)$. (B) PL decay curve of benzoic acid derivatives at $\lambda_{\mathrm{ex}}=365 \mathrm{~nm}$, $\lambda_{\mathrm{em}}=500 \mathrm{~nm}$. PL spectra of (C) $o$ MOBA crystal and (D) $o$ DMOBA crystal with delay of $0 \mathrm{~ms}$ (blue spectra) and $10 \mathrm{~ms}$ (green spectra) at room temperature under ambient condition, $\lambda_{\mathrm{ex}}=$ $285 \mathrm{~nm}$. Inset: the average lifetimes of delayed fluorescence at $\lambda_{\mathrm{em}}=360 \mathrm{~nm}$ and phosphorescence at $\lambda_{\mathrm{em}}=500 \mathrm{~nm}$. Photographs taken before and after the removal of a $365 \mathrm{~nm}$ UV lamp. 


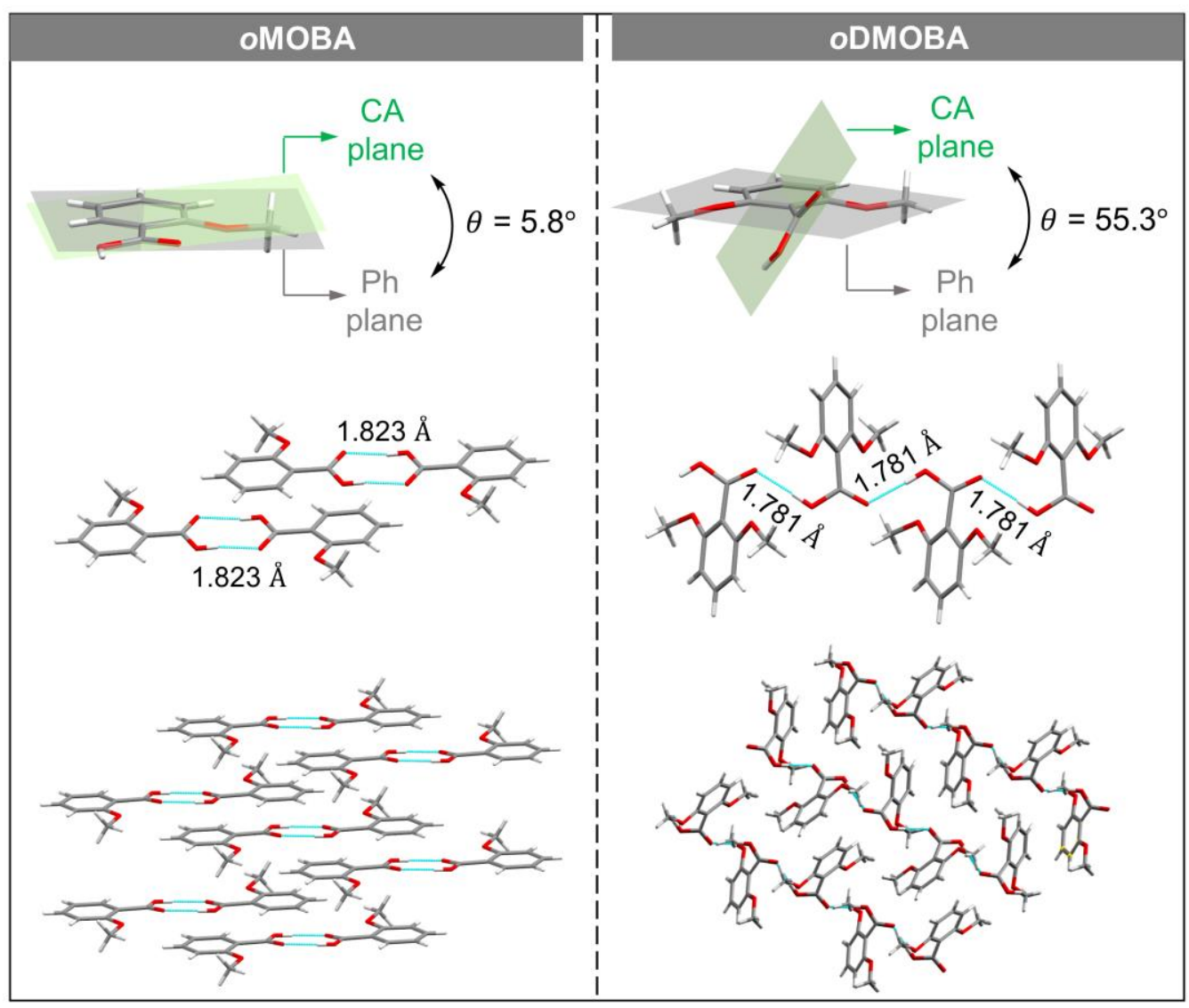

Figure 2. Crystal structures and packing patterns of $o$ MOBA and $o$ DMOBA. $\theta$ represents the dihedral angle between the carboxylic acid (CA) plane and the Phenyl (Ph) plane. Blue dash lines represent intramolecular hydrogen bonds. 

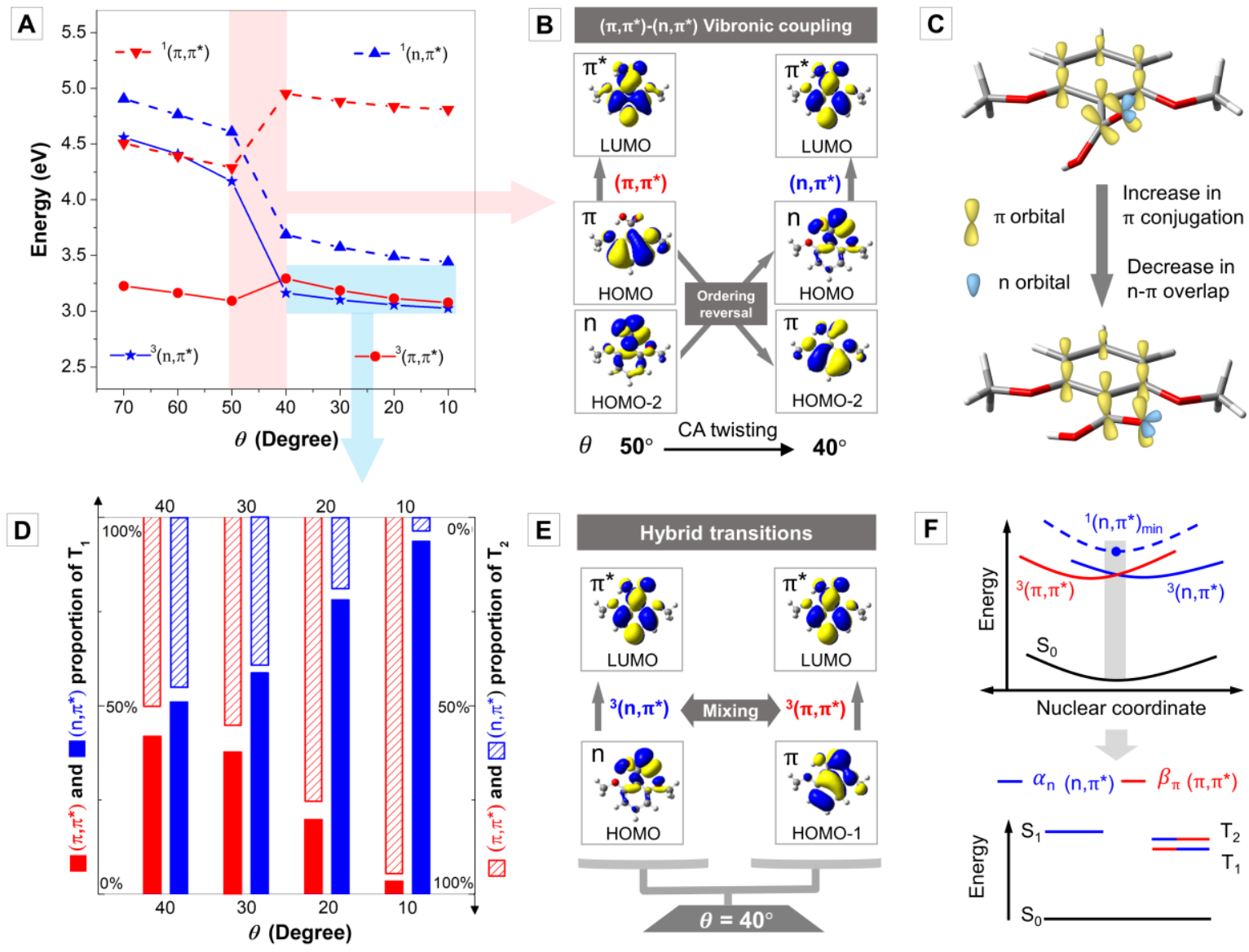

Figure 3. (A) PESs of the first two excited states in both singlet and triplet manifolds of isolated $o$ DMOBA molecule along the axis of CA twisting at the geometries of $\mathrm{S}_{1, \min }$. (B) The FMOs at the twisting angles of $40^{\circ}$ and $50^{\circ}$. (C) Schematic illustration of orbital overlap change between the $\pi$ plane and the $n$ orbital on the carbonyl oxygen along with the CA twisting. (D) The proportions of ${ }^{3}\left(n, \pi^{*}\right)$ and ${ }^{3}\left(\pi, \pi^{*}\right)$ transitions in hybrid $T_{1}$ and $T_{2}$ at the twisting angle from $10^{\circ}$ to $40^{\circ}$. (E) The FMOs of hybrid transitions and (F) Schematic PESs (up) and Jablonski diagram (down) at the geometry of ${ }^{1}\left(\mathrm{n}, \pi^{*}\right)_{\min }$ at the twisting angle of $40^{\circ}$, The proportions of ${ }^{3}\left(\mathrm{n}, \pi^{*}\right)$ and ${ }^{3}\left(\pi, \pi^{*}\right)$ are named as $\alpha_{\mathrm{n}}$ or $\beta_{\pi}$, respectively. 

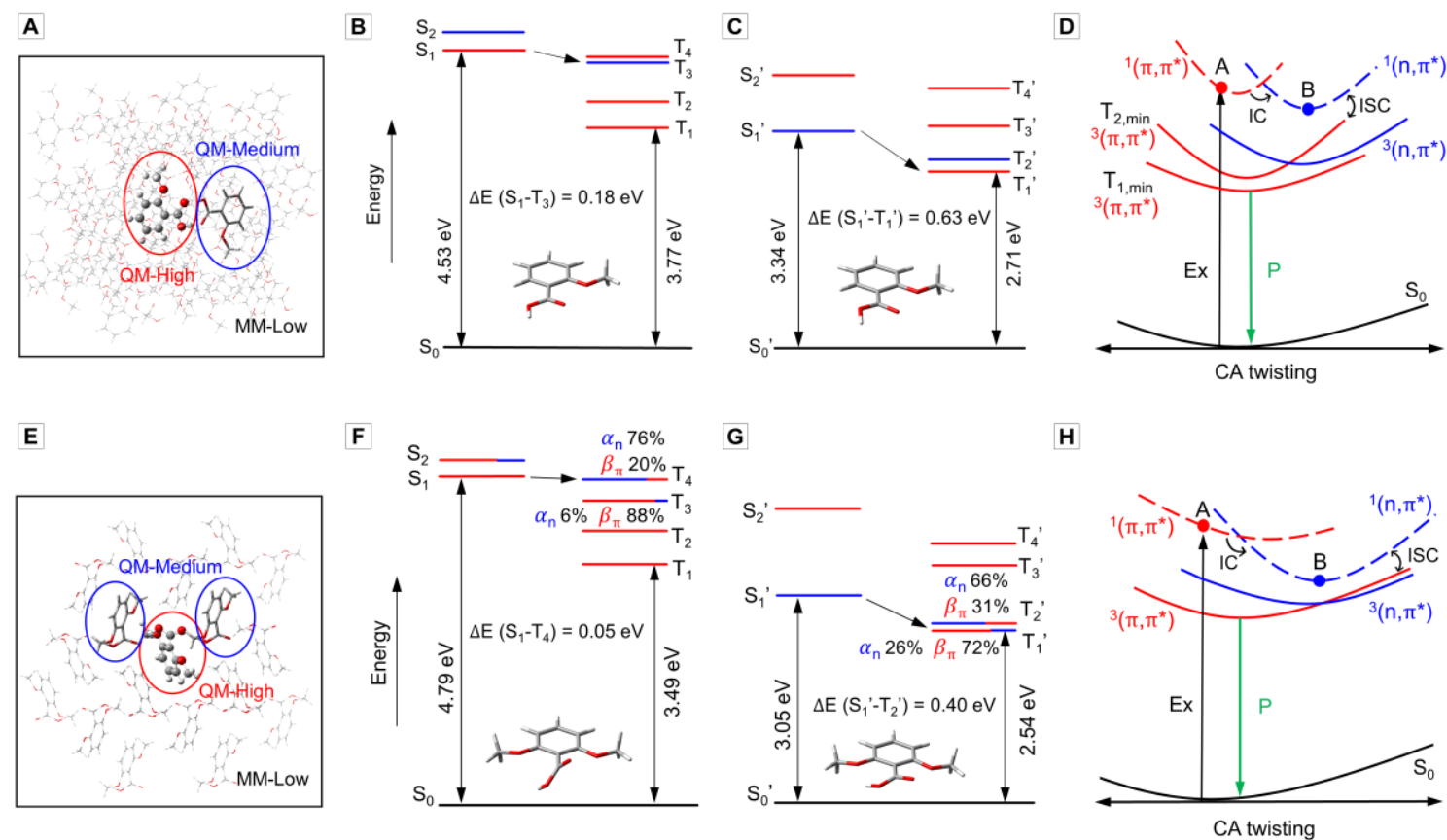

Figure 4. Setup of three-layer model of (A) $o$ MOBA and (E) $o$ DMOBA crystals. Energy level diagrams of $(\mathrm{B}, \mathrm{C}) o \mathrm{MOBA}$ and $(\mathrm{F}, \mathrm{G}) o \mathrm{DMOBA}$ at the geometries of $(\mathrm{B}, \mathrm{F})$ Frank-Condon point (i.e. point A of Fig. D,H) and $(\mathrm{C}, \mathrm{G})^{1}\left(\mathrm{n}, \pi^{*}\right)_{\min }$ (i.e. point B of Fig. D,H). Insets: corresponding molecular structures. Energy levels drawn as pure blue or red bars have $\alpha_{\mathrm{n}}$ or $\beta_{\pi}$ proportions larger than 95\%. Schematic PESs of (D) $o$ MOBA and (G) $o$ DMOBA crystals, Abbreviation: $\mathrm{IC}=$ internal conversion, $\mathrm{ISC}=$ intersystem crossing, $\mathrm{Ex}=$ excitation, $\mathrm{P}=$ phosphorescence.

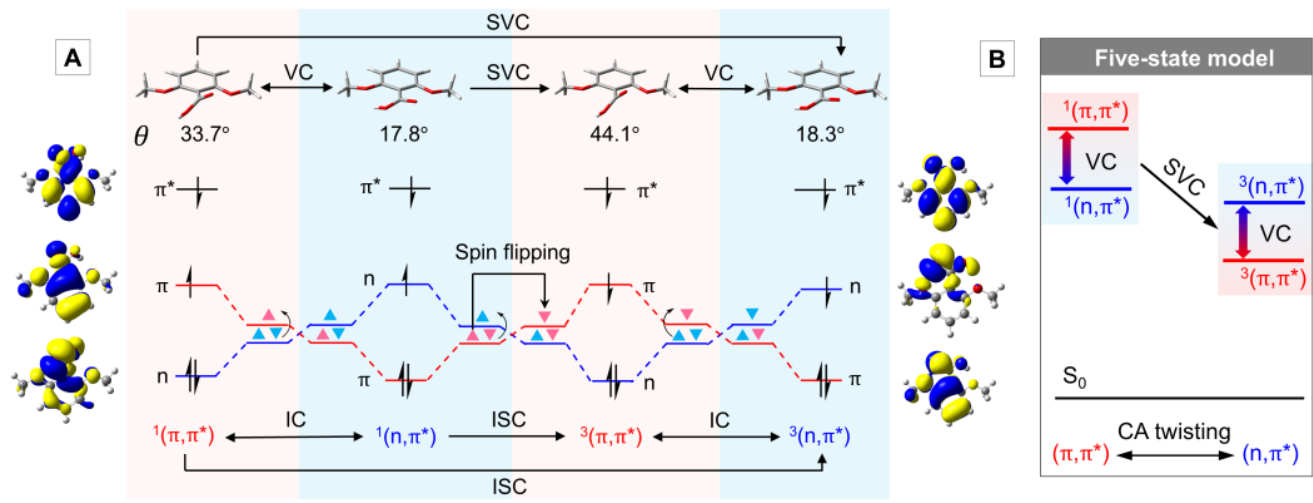

Figure 5. (A) Optimized structures and FMO diagrams at the minima of ${ }^{1}\left(\pi, \pi^{*}\right),{ }^{1}\left(\mathrm{n}, \pi^{*}\right),{ }^{3}\left(\pi, \pi^{*}\right)$, and ${ }^{3}\left(n, \pi^{*}\right)$ excited states. (B) Schematic illustration of the five-state model. Abbreviation: VC $=$ vibronic coupling, $\mathrm{SVC}=$ spin vibronic coupling, $\mathrm{IC}=$ internal conversion, $\mathrm{ISC}=$ intersystem crossing. 


\section{Supporting Information}

\section{The Importance of Solid-state Molecular Motion to Room Temperature Phosphorescence}

Yujie Tu, Junkai Liu, Xuepeng Zhang, Tsz Shing Cheung, Xuewen He, Jing Guo, Jacky W.Y. Lam, Sijie Chen, * Ben Zhong Tang*

Dr. Y. Tu, J. Liu, Dr. X. Zhang, T.S.Cheung, Dr. X. He, Prof. J.W.Y.Lam, Prof. B.Z.Tang. Department of Chemistry, Hong Kong Branch of Chinese National Engineering Research Center for Tissue Restoration and Reconstruction, State Key Laboratory of Neuroscience, Department of Chemical and Biological Engineering, and Division of Life Science, The Hong Kong University of Science and Technology, Clear Water Bay, Kowloon, 999077, Hong Kong, China. Center for Aggregation-Induced Emission, SCUT-HKUST Joint Research Institute, State Key Laboratory of Luminescent Materials and Devices, South China University of Technology, Guangzhou, 510640, China. E-mail: tangbenz@ust.hk

Dr. S. Chen, Prof. B.Z.Tang. Ming Wai Lau Centre for Reparative Medicine, Karolinska Institutet, Hong Kong. E-mail: sijie.chen@ki.se

Prof. B.Z.Tang, AIE Institute, Guangzhou Development District, Huangpu, Guangzhou 510530, China.

J. Guo. State Key Laboratory of Chemo/Biosensing and Chemometrics, College of Chemistry and Chemical Engineering, Hunan University, Changsha 410082, China.

\section{Methods}

\section{(1) Materials and instrumentations}

The benzoic acid (BA), 4-methoxybenzoic acid ( $p$ MOBA), 3-methoxybenzoic acid ( $m$ MOBA), 2-methoxybenzoic acid (oMOBA), and 2,6-dimethoxybenzoic acid (oDMOBA) were purchased from Meryer Co., Ltd. The crystals were obtained by recrystallization in dichloromethane/n-hexane mixture for three times. UV-vis absorption spectra were recorded on a Varian Cary 50 UV-visible spectrophotometer. Prompt and delayed photoluminescence spectra and the fluorescence lifetimes were measured using an Edinburgh FLSP920 fluorescence spectrophotometer. The quantum yield values were measured on Quantaurus-QY Plus absolute PL quantum yield spectrometer C13534. 


\section{(2) Calculation method}

The crystal structures are retrived from the CCDC database: $\mathrm{BA}(787428), p \mathrm{MOBA}(751233)$, $m$ MOBA(1448794), $o$ MOBA(1047316), oDMOBA (722066). The PESs of $o$ DMOBA in gas phase were calculated in the Gaussian 09 package at the (TD)PBE0/6-311G** level. The $\mathrm{S}_{0}$ and $\mathrm{S}_{1}$ of $o$ DMOBA with fixed CA twisting angle from $10^{\circ}$ to $70^{\circ}$ were optimized. As for crystals, a three-layer ONIOM model with the embedded charge mode based on the single crystal structures. The central molecule is treated as the high-layer QM part at (TD)PBE0/6$311 \mathrm{G}^{* *}$ level for both $o$ MOBA and $o$ DMOBA. The adjacent one oMOBA molecule or two oDMOBA molecules which interact with the central molecules via hydrogen bonding are treated as the middle-layer QM part at PBE0/6-311G** level. The other surrounding molecules are treated as the MM part using the universal force field (UFF). We also conduct frequency calculation to confirm all the steady points are at the lowest energy. The computational results (Table S5 and S7) are consistent with the experimental data (Fig. 1C and 1D), indicating the (TD)PBE0/6-311G** method is appropriate for the simulations in this work.

\section{Supplementary experimental results}

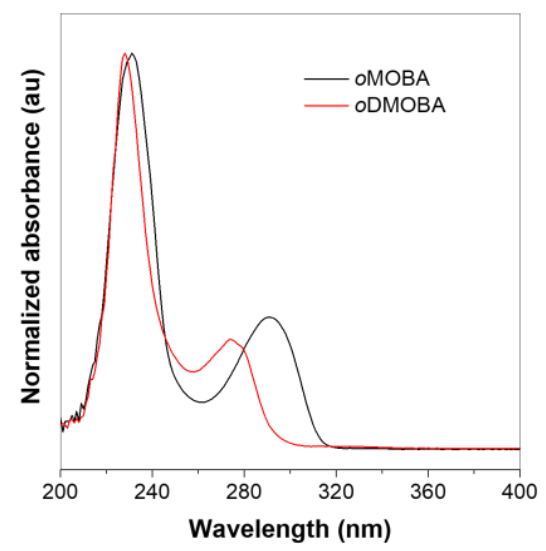

Figure S1. Absorption spectra of $o \mathrm{MOBA}$ and $o \mathrm{DMOBA}$ in THF solution. 

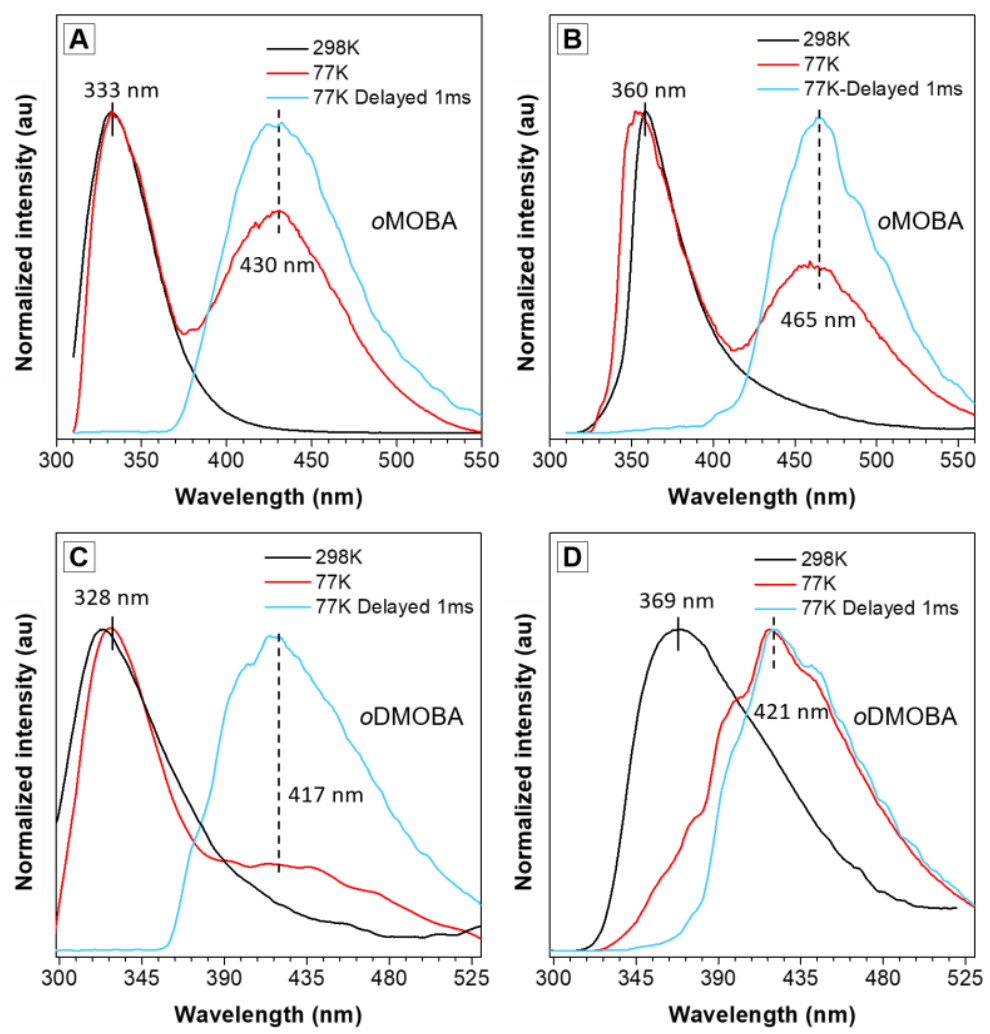

Figure S2. PL spectra of (A and B) $o$ MOBA (C and D) $o$ DMOBA in (A and C) THF solution and (B and D) in the crystalline state at $298 \mathrm{~K}, 77 \mathrm{~K}$ with delay of 0 and $1 \mathrm{~ms}$.
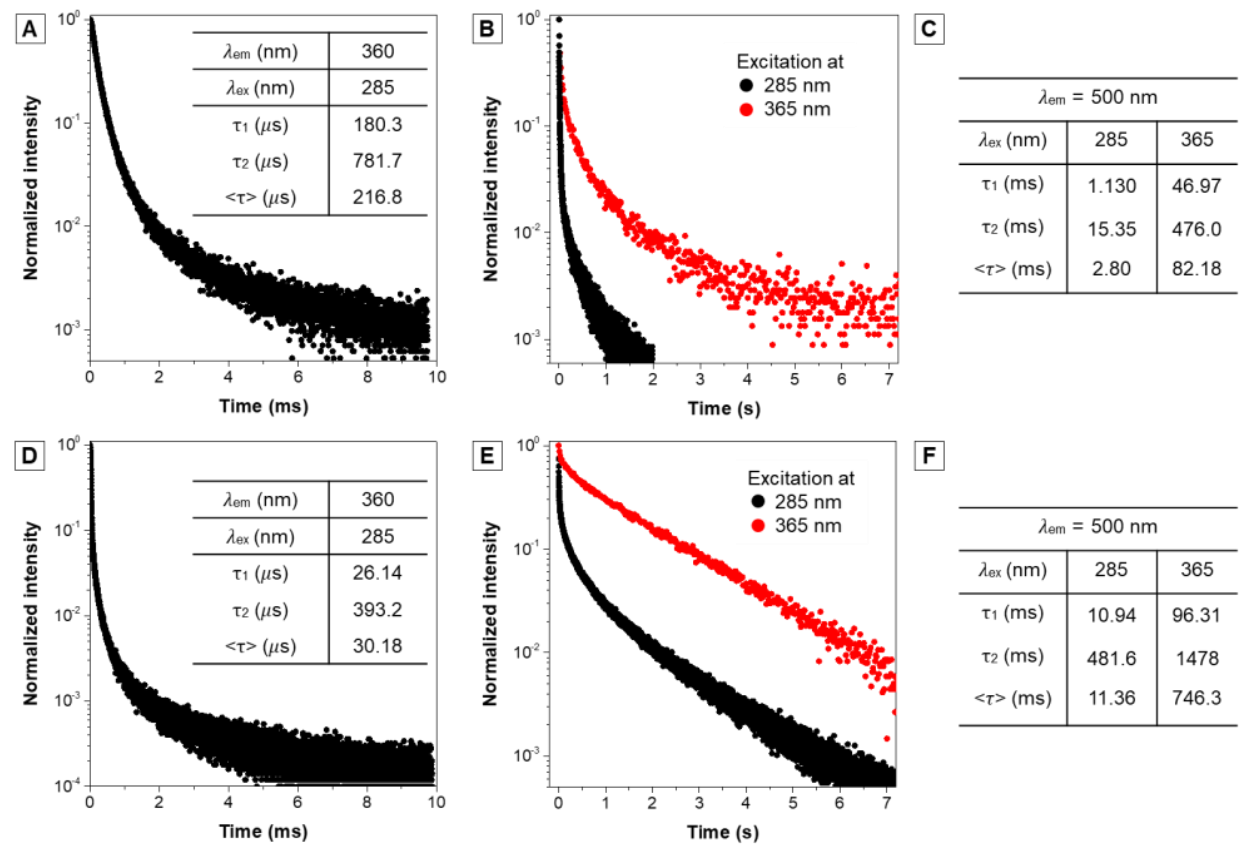

\begin{tabular}{c|c|c}
\hline \multicolumn{3}{c}{$\lambda_{e m}=500 \mathrm{~nm}$} \\
\hline$\lambda_{\text {ex }}(\mathrm{nm})$ & 285 & 365 \\
\hline$\tau_{1}(\mathrm{~ms})$ & 10.94 & 96.31 \\
$\tau_{2}(\mathrm{~ms})$ & 481.6 & 1478 \\
$<\tau>(\mathrm{ms})$ & 11.36 & 746.3 \\
\hline
\end{tabular}

Figure S3. PL decay curves of (A) $o$ MOBA and (D) $o$ DMOBA at the $\lambda_{\mathrm{em}}=360 \mathrm{~nm}$, Inset: the corresponding lifetime values. PL decay curves of (B) $o$ MOBA and (E) $o$ DMOBA at the $\lambda_{\mathrm{em}}=$ $500 \mathrm{~nm}$. The lifetime values of (C) $o$ MOBA and (F) $o$ DMOBA at $\lambda_{\mathrm{em}}=500 \mathrm{~nm}$. 
Table S1. Quantum yields of $o \mathrm{MOBA}$ and $o \mathrm{DMOBA}$

\begin{tabular}{lcc}
\hline Excitation wavelength & $o$ MOBA & $o$ DMOBA \\
\hline $285 \mathrm{~nm}-\left(\pi, \pi^{*}\right)$ excitation & $10.8 \%$ & $5.7 \%$ \\
$365 \mathrm{~nm}-\left(\mathrm{n}, \pi^{*}\right)$ excitation & $2.7 \%$ & $13.0 \%$ \\
\hline
\end{tabular}

BA
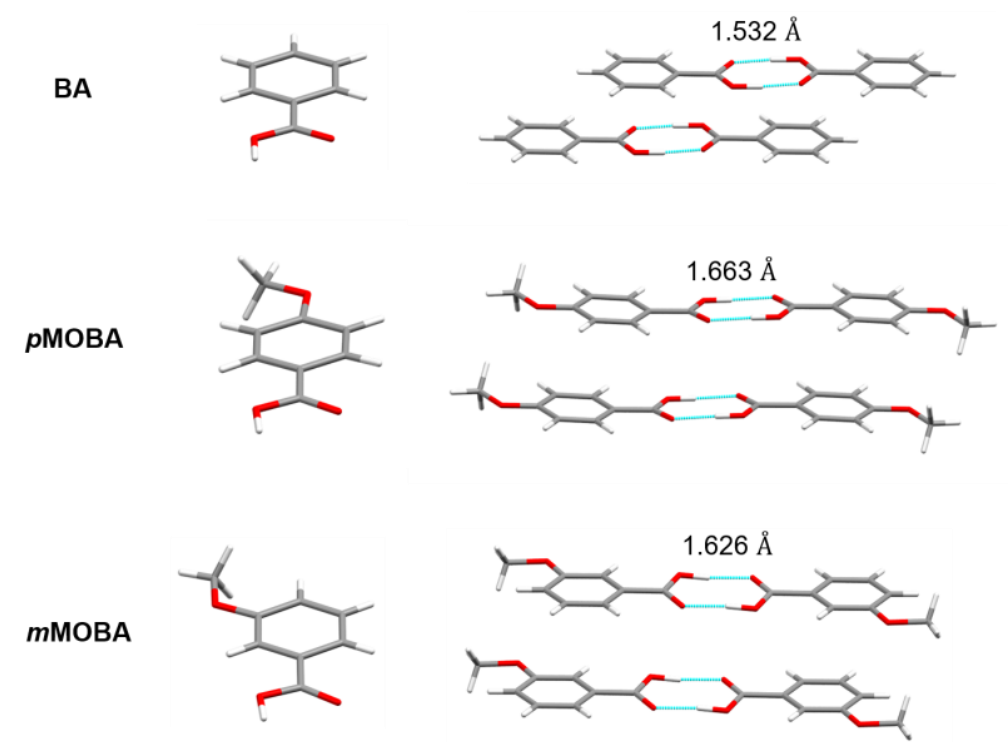

Figure S4. Crystal structures and packing patterns of BA, $p$ MOBA and $m \mathrm{MOBA}$. Blue dash lines represent intramolecular hydrogen bonds.

\section{Calculation results}

\section{Crystal structure}

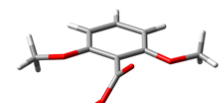

$\theta=55.3^{\circ}$

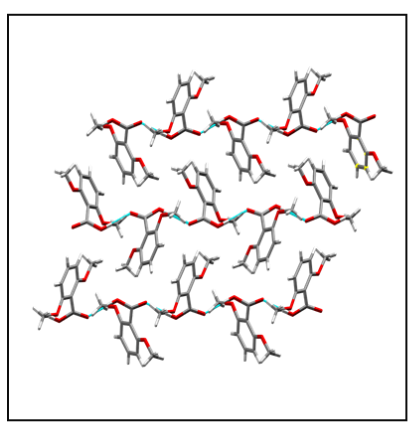

Optimized ground state
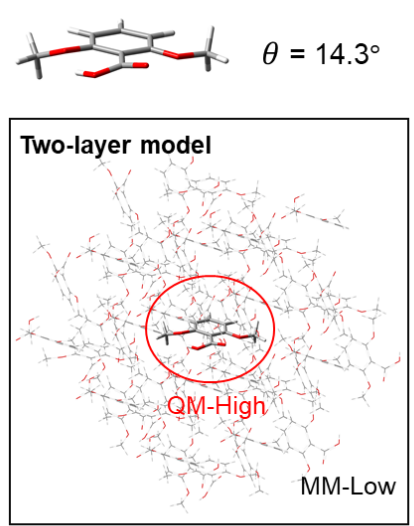

$\theta=52.3^{\circ}$

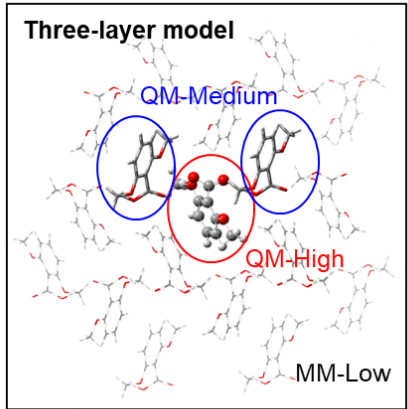

Figure S5. The crystal structure of $o$ DMOBA and optimized ground states of $o$ DMOBA adopting two-layer and three-layer models. 
Table S2. FMOs of isolated $o$ DMOBA at the $\mathrm{S}_{1, \min }$ geometries with different fixed $\theta$.

\begin{tabular}{|c|c|c|c|c|}
\hline$\theta$ & HOMO-2 & HOMO-1 & HOMO & LUMO \\
\hline \multicolumn{5}{|l|}{$10^{\circ}$} \\
\hline \multicolumn{5}{|l|}{$20^{\circ}$} \\
\hline \multicolumn{5}{|l|}{$30^{\circ}$} \\
\hline \multicolumn{5}{|l|}{$40^{\circ}$} \\
\hline \multicolumn{5}{|l|}{$50^{\circ}$} \\
\hline \multicolumn{5}{|l|}{$60^{\circ}$} \\
\hline $70^{\circ}$ & & & & \\
\hline
\end{tabular}

Table S3. Transition information of first two excited states of both singlet and triplet manifolds of isolated $o$ DMOBA at the $\mathrm{S}_{1, \min }$ geometries with fixed $\theta=40^{\circ}$ and $50^{\circ}$.

\begin{tabular}{cccccc}
\hline $\boldsymbol{\theta}$ & $\begin{array}{c}\text { Excited } \\
\text { state }\end{array}$ & $\begin{array}{c}\text { Vertical } \\
\text { transition } \\
(\mathrm{eV})\end{array}$ & $\begin{array}{c}\text { Wavelength } \\
(\mathrm{nm})\end{array}$ & \multicolumn{1}{c}{ Transitions } & $\begin{array}{c}\text { Oscillator } \\
\text { strength }\end{array}$ \\
\hline $\mathrm{S}_{1, \mathrm{~min}}$ & 3.10 & 400 & $\mathrm{H} \rightarrow \mathrm{L}^{1}\left(\mathrm{n}, \pi^{*}\right) 93.8 \%$ & 0.0010 \\
\hline $\mathrm{S}_{2}$ & 4.36 & 284 & $\begin{array}{l}\mathrm{H}-2 \rightarrow \mathrm{L}^{1}\left(\pi, \pi^{*}\right) 51.2 \% \\
\mathrm{H}-1 \rightarrow \mathrm{L}^{1}\left(\pi, \pi^{*}\right) 40.4 \%\end{array}$ & 0.0956 \\
\cline { 2 - 6 } & 2.58 & 481 & $\begin{array}{l}\mathrm{H}-1 \rightarrow \mathrm{L}^{3}\left(\pi, \pi^{*}\right) 35.6 \% \\
\mathrm{H} \rightarrow \mathrm{L}^{3}\left(\mathrm{n}, \pi^{*}\right) 51.0 \%\end{array}$ & 0.0000 \\
\hline $\mathrm{T}_{1}$ & 2.71 & 458 & $\begin{array}{l}\mathrm{H}-1 \rightarrow \mathrm{L}^{3}\left(\pi, \pi^{*}\right) 44.4 \% \\
\mathrm{H} \rightarrow \mathrm{L}^{3}\left(\mathrm{n}, \pi^{*}\right) 39.3 \%\end{array}$ & 0.0000 \\
\hline $\mathrm{T}_{2}$ & 4.29 & 289 & $\mathrm{H} \rightarrow \mathrm{L}^{1}\left(\pi, \pi^{*}\right) 90.2 \%$ & 0.0811 \\
\hline $\mathrm{S}_{1, \min }$ & 4.61 & 269 & $\begin{array}{l}\mathrm{H}-2 \rightarrow \mathrm{L}^{1}\left(\mathrm{n}, \pi^{*}\right) 73.8 \% \\
\mathrm{H}-1 \rightarrow \mathrm{L}^{1}\left(\pi, \pi^{*}\right) 15.9 \%\end{array}$ & 0.0129 \\
\hline $\mathrm{S}_{2}$ & 3.09 & 401 & $\begin{array}{l}\mathrm{H}-1 \rightarrow \mathrm{L}^{3}\left(\pi, \pi^{*}\right) 62.3 \% \\
\mathrm{H} \rightarrow \mathrm{L}^{3}\left(\pi, \pi^{*}\right) 18.9 \%\end{array}$ & 0.0000 \\
\hline $\mathrm{T}_{1}$ & 4.16 & 298 & $\begin{array}{l}\mathrm{H}-2 \rightarrow \mathrm{L}^{3}\left(\mathrm{n}, \pi^{*}\right) 61.8 \% \\
\mathrm{H} \rightarrow \mathrm{L}+13\left(\pi, \pi^{*}\right) 16.0 \%\end{array}$ & 0.0000 \\
\hline
\end{tabular}


Table S4. FMOs of $o$ MOBA in crystal at different electronic states.

Structure

Table S5. Transition information of $o \mathrm{MOBA}$ in crystal at different electronic states.

\begin{tabular}{cccccc}
\hline $\begin{array}{c}\text { Electronic } \\
\text { state }\end{array}$ & $\begin{array}{c}\text { Adiabatic } \\
\text { transition } \\
(\mathrm{eV})\end{array}$ & $\begin{array}{c}\text { Vertical } \\
\text { transition } \\
(\mathrm{eV})\end{array}$ & $\begin{array}{c}\text { Wavelength } \\
(\mathrm{nm})\end{array}$ & Transition & $\begin{array}{c}\text { Oscillator } \\
\text { strength }\end{array}$ \\
\hline $\mathrm{S}_{1}, \mathrm{FC}$ point & 4.53 & 4.53 & 273 & $\mathrm{H} \rightarrow \mathrm{L}(91.6 \%)$ & 0.1029 \\
\hline $1\left(\pi, \pi^{*}\right)_{\min }$ & 4.34 & 4.14 & 299 & $\mathrm{H} \rightarrow \mathrm{L}(94.3 \%)$ & 0.1058 \\
\hline $1\left(\mathrm{n}, \pi^{*}\right)_{\min }$ & 4.13 & 3.34 & 371 & $\begin{array}{l}\mathrm{H}-1 \rightarrow \mathrm{L} \\
(93.8 \%)\end{array}$ & 0.0001 \\
\hline $\begin{array}{c}\mathrm{T}_{2, \min } \\
\left(\pi, \pi^{*}\right)\end{array}$ & 2.99 & 2.65 & 468 & $\mathrm{H} \rightarrow \mathrm{L}(88.2 \%)$ & 0.0000 \\
\hline $\begin{array}{c}\mathrm{T}_{1, \min } \\
\left(\pi, \pi^{*}\right)\end{array}$ & 2.99 & 2.59 & 479 & $\mathrm{H} \rightarrow \mathrm{L}(84.7 \%)$ & 0.0000 \\
\hline
\end{tabular}


Table S6. FMOs of $o$ DMOBA in crystal at different electronic states.

(n)

Table S7. Transition information of $o$ DMOBA in crystal at different electronic states.

\begin{tabular}{cccccc}
\hline $\begin{array}{c}\text { Electronic } \\
\text { states }\end{array}$ & $\begin{array}{c}\text { Adiabatic } \\
\text { transition }(\mathrm{eV})\end{array}$ & $\begin{array}{c}\text { Vertical } \\
\text { transition } \\
(\mathrm{eV})\end{array}$ & $\begin{array}{c}\text { Wavelength } \\
(\mathrm{nm})\end{array}$ & Transitions & $\begin{array}{c}\text { Oscillator } \\
\text { strength }\end{array}$ \\
\hline $\mathrm{S}_{1}, \mathrm{FC}$ Point & 4.79 & 4.79 & 259 & $\begin{array}{l}\mathrm{H} \rightarrow \mathrm{L}(82.5 \%) \\
\mathrm{H} \rightarrow \mathrm{L}+1\end{array}$ & 0.0601 \\
\hline${ }^{1}\left(\pi, \pi^{*}\right)_{\min }$ & 5.09 & 4.71 & 263 & $\begin{array}{l}\mathrm{H}-2 \rightarrow \mathrm{H}-1 \rightarrow \mathrm{L}(24.4 \%) \\
\mathrm{H} \rightarrow \mathrm{L}(49.4 \%)\end{array}$ & 0.1159 \\
\hline${ }^{1}\left(\mathrm{n}, \pi^{*}\right)_{\min }$ & 4.13 & 3.05 & 406 & $\mathrm{H} \rightarrow \mathrm{L}(95.4 \%)$ & 0.0005 \\
\hline${ }^{3}\left(\mathrm{n}, \pi^{*}\right)_{\min }$ & 3.71 & 2.59 & 479 & $\mathrm{H} \rightarrow \mathrm{L}(84.0 \%)$ & 0.0000 \\
\hline${ }^{3}\left(\pi, \pi^{*}\right)_{\min }$ & 3.07 & 2.53 & 489 & $\mathrm{H} \rightarrow \mathrm{L}(92.2 \%)$ & 0.0000 \\
\hline
\end{tabular}

